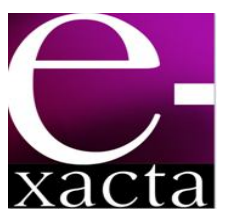

ISSN: 1984-3151

\title{
ESTERIFICAÇÃO HOMOGÊNEA DOS ÁCIDOS GRAXOS LIVRES DO ÓLEO DA POLPA DE MACAÚBA (ACROCOMIA ACULEATA)
}

\author{
HOMOGENEOUS ESTERIFICATION OF FREE FATTY ACID FROM
} MACAUBA PULP OIL (ACROCOMIA ACULEATA) \author{
Silva ${ }^{4}$ \\ 1 Mestranda em Bioenergia, Universidade Estadual de \\ Maringá. Umuarama, PR. Tecnóloga em Alimentos, \\ Universidade Estadual de Maringá, PR. 2012. \\ djessicaraspe@hotmail.com. \\ 2 Graduanda de Química Industrial, Universidade \\ Paranaense. Umuarama, 2013. \\ brunamellosjp@hotmail.com. \\ 3 Graduando em Química Industrial, Universidade \\ Paranaense. Umuarama, 2013. \\ pablo.jorge.silva@gmail.com. \\ 4 Doutora em Engenharia Química. Universidade Estadual de \\ Maringá, 2009. Professora do Departamento de Tecnologia. \\ Universidade Estadual de Maringá, Umuarama, PR. \\ camiladasilva.eq@gmail.com.
}

Djéssica Tatiane Raspe ${ }^{1}$; Bruna Tais Ferreira de Mello²; Pablo Jorge da Silva ${ }^{3}$; Camila da

Recebido em: 19/09/2013 - Aprovado em: 30/04/2014 - Disponibilizado em: 31/05/2014

RESUMO: O presente trabalho buscou avaliar a utilização do ácido sulfúrico $\left(\mathrm{H}_{2} \mathrm{SO}_{4}\right)$ como catalisador na reação de esterificação dos ácidos graxos livres (AGLs) do óleo da polpa de Macaúba utilizando metanol e etanol. Os experimentos avaliaram o efeito do percentual de catalisador, razão molar AGLs:álcool, temperatura e tempo de reação. Os resultados obtidos mostram que o metanol é mais eficiente na esterificação dos AGLs, quando comparado ao etanol. Verificou-se que o aumento do percentual de catalisador influenciou significativamente no aumento da conversão da reação, para ambos os alcoóis. Avaliou-se o efeito da razão molar (AGL:álcool) de 1:1; 1:6; 1:9; 1:12; 1:15 e 1:18, sendo verificadas para o etanol conversões de $61 \%$ sob a razão molar 1:9, e conversões na ordem de 93\% foram obtidas sob razão molar 1:18 para o metanol. Em razão molar AGLs:metanol $1: 18,2 \%$ de catalisador e 60 minutos de reação, verificou-se que o aumento da temperatura reacional promoveu aumento na conversão dos $A G L s$ na faixa de $30{ }^{\circ} \mathrm{C}$ a $50 \stackrel{\circ}{\circ}$.

PALAVRAS-CHAVE: Esterificação. Óleo da polpa de Macaúba. Ácidos Graxos Livres.

ABSTRACT: This study evaluated the use of sulfuric acid $\left(\mathrm{H}_{2} \mathrm{SO}_{4}\right)$ as catalysts for the esterification of free fatty acids (FFAs) from Macauba pulp oil using methanol and ethanol. The experiments evaluated the effect of the percentage of catalyst, molar ratio FFA: alcohol, temperature and reaction time. The results show that methanol is more efficient for esterification of free fatty acids when compared to ethanol. It was found that increasing the percentage of catalyst increased influenced significantly the conversion reaction, for both alcohols. We evaluated the effect of molar ratio (FFA: alcohol) of 1:1, 1:6, 1:9, 1:12, 1:15 and 1:18, and checked for ethanol conversion of $21 \%$ in the molar ratio of 1:9, and conversions in the order of $93 \%$ have been obtained using molar ratio of 1:18 for methanol. In molar ratio FFA:methanol of 1:18, 2\% catalyst and 60 minutes of reaction, it was found that the increase in reaction temperature increased the conversion of free fatty acids in the range $30^{\circ} \mathrm{C}$ to $50{ }^{\circ} \mathrm{C}$.

KEYWORDS: Esterification. Macauba pulp oil. Free fatty acid. 


\section{INTRODUÇÃO}

As pesquisas sobre combustíveis alternativos sustentáveis têm sido amplamente priorizadas ao redor do mundo, principalmente as que visem a substituir os combustíveis fósseis por intermédio do biodiesel. Considerado o substituto mais atraente do diesel de origem fóssil, o biodiesel traz como vantagem seu custo relativamente menor, a compatibilidade com a atual infraestrutura do diesel e a disponibilidade da tecnologia de produção (NG; GAN, 2010). Além disso, estudos dos mesmos autores envolvendo a combustão do biodiesel em motores do ciclo diesel demonstram-no um potente redutor das emissões de poluentes.

Segundo a Lei ㄲo 11.097, de 13 de Janeiro de 2005, vigente no Brasil, biodiesel é um "combustível derivado de biomassa renovável para uso em motores à combustão interna com ignição por compressão, ou, conforme regulamento, para geração de outro tipo de energia que possa substituir parcial ou totalmente combustíveis de origem fóssil" (Portal do Biodiesel Brasil). É um combustível não tóxico, biodegradável derivado de fontes renováveis e com crescente disponibilidade (McCORNICK et al., 2001), o que justifica o crescente interesse pelo desenvolvimento de tecnologias referentes à sua produção.

O biodiesel pode ser produzido através da reação de transesterificação, a qual ocorre entre óleos vegetais ou gorduras animais na presença de um álcool e, geralmente, um catalisador alcalino (GAN et al., 2012). Óleos vegetais refinados, incluindo o óleo de canola e o óleo de soja, são comumente usados como matérias-primas para a produção deste biocombustível. No entanto, para o biodiesel produzido a partir de óleos refinados, o custo de matéria-prima contribui com mais de $70 \%$ dos custos totais de produção (ZULLAIKAH et al., 2005; LOU; ZONG; DUAN, 2008), o que representa um grande desafio na comercialização e seu uso generalizado do
(NG; GAN, 2010). Além disso, a indústria deste produto tem enfrentado constante pressão em função da possível disputa das terras aráveis, "alimentos versus combustível" (GAN et al., 2012).

Recentemente, intensificam-se os incentivos para a utilização de demais substratos visando à utilização de uma matriz oleaginosa sem fins alimentícios. Uma fonte alternativa para produção de biodiesel é o óleo do fruto da Macaúba (Acrocomia aculeata), devido ao grande potencial para produção de óleo, 1500 a 5000 litros de óleo por hectare. A Acrocomia aculeata produz óleo de alta qualidade e é considerada uma matriz oleaginosa com grande potencial para a produção sustentável de biocombustíveis (ABREU et al., 2011), destacando-se sob os aspectos ambiental, econômico e social.

O óleo de macaúba apresenta elevado percentual de ácidos graxos livres (entre 10 a $70 \%$ ) e água (>0,5 $\%$ ), tornando o processo convencional a partir da transesterificação com catalisador homogêneo alcalino inviável, visto que para este processo o percentual de ácidos graxos livres e água deve ser inferior a $0,5 \%$ e $0,05 \%$ respectivamente (VYAS; VERMA; SUBRAHMANYAM, 2010). O alto conteúdo de ácidos graxos livres ocasiona a formação de sabões, o que reduz o rendimento da reação e dificulta o processo de separação e purificação dos produtos (MARCHETTI; MIGUEL; ERRAZU, 2007; TALUKDER; WU; CHUA, 2010), significando altos custos de produção e energia (RAMOS et al., 2003).

A esterificação é uma rota alternativa, indicada quando o teor de ácidos graxos livres na matériaprima é alto, ou mesmo para aproveitamento de resíduos de ácidos graxos (LOTERO et al., 2005; MA; HANNA, 1999; DI SERIO et al., 2008). Apresenta taxas de reação superiores à transesterificação e permite 0 uso de catalisadores ácidos sem a ocorrência de saponificação (MBARAKA et al., 2003). Estudos recentes destacam a proposta do processo 
de obtenção de ésteres de ácidos graxos em duas etapas para substratos com elevada acidez. A proposta consiste na esterificação dos ácidos graxos livres e posterior transesterificação dos triglicerídeos (RAMADHAS; JAYARAJ; MURALEEDHARAN, 2005; SUAREZ et al., 2007; BERRIOS et al., 2010; SRILATHA et al., 2010; CORRO et al., 2011) ou a aplicação do processo de Hidroesterificação (MINAMI; SAKA, 2006; GAN et al., 2010; ROCHA et al., 2010; SOUSA et al., 2010; TALUKDER; WU; CHUA, 2010) Dentro deste contexto, o presente trabalho teve por objetivo o estudo da reação de esterificação homogênea dos ácidos graxos livres (AGLs) do óleo da polpa da Macaúba, avaliando os efeitos das variáveis de processo percentual de catalisador, razão molar AGL:álcool, temperatura e tempo de reação. Com este estudo, busca-se reduzir o teor de AGLs no óleo de Macaúba, obtendo um óleo vegetal que poderá ser utilizado para a etapa de transesterificação alcalina convencional.

\section{MATERIAIS E MÉtodos}

\subsection{MATERIAIS}

Para as reações foram utilizados como substratos o óleo da polpa da Macaúba (Cocal - Brasil), álcool metílico (Merck 99,9\% de pureza) e etílico (Merck 99,9\% de pureza). A composição em ácidos graxos do óleo de Macaúba é apresentada na Tabela 1, sendo que o percentual de ácidos graxos livres foi de $71,1 \pm 0,4 \%$ e o teor de água de $0,10 \pm 0,1 \%$. Como catalisador, foi utilizado o ácido sulfúrico (Anidrol $99,9 \%$ de pureza). Na etapa de titulação das amostras, foram utilizados: solução de etanol/éter etílico 1:2 (v:v) (Vetec/Nuclear), hidróxido de sódio 0,1 $\mathrm{N}$ (Nuclear) e o indicador fenolftaleína (Nuclear).
Tabela 1

Percentual de ácidos graxos totais do óleo de Macaúba

\begin{tabular}{cc}
\hline Ácidos Graxos & $(\%)$ \\
\hline Cáprico C10:0 & $0,13 \pm 0,004$ \\
Láurico C12:0 & $1,42 \pm 0,008$ \\
Mirístico C14:0 & $0,45 \pm 0,008$ \\
Palmítico C16:0 & $13,31 \pm 0,013$ \\
Palmitoléico C16:1n-7 & $1,38 \pm 0,005$ \\
Esteárico C18:0 & $2,81 \pm 0,035$ \\
Oléico C18:1n9 & $64,84 \pm 0,076$ \\
cis-vaccênico C18:1n7 & $2,34 \pm 0,013$ \\
Linoléico C18:2n-6 & $7,77 \pm 0,040$ \\
Linolênico C18:3n-3 & $0,27 \pm 0,002$ \\
Araquídico C20:0 & $0,24 \pm 0,006$ \\
Eicosadienoico C20:2n6 & $0,16 \pm 0,008$ \\
Não Identificado & $4,88 \pm 0,021$ \\
\hline
\end{tabular}

\subsection{REAÇõES DE ESTERIFICAÇÃo}

As reações foram conduzidas em um balão de três bocas, onde se pesou o óleo de Macaúba. Conectouse o balão a um condensador e a uma chapa de aquecimento com controle de temperatura e agitação, conforme esboçado na Figura 1. Paralelamente pesou-se o álcool e o catalisador em um béquer. Após a temperatura de reação ser alcançada, adicionou-se a mistura álcool-catalisador e esperou-se o tempo de reação. Dado o tempo reacional, transferiu-se a mistura reacional para um balão de separação, onde esta mesma foi lavada com água destilada na temperatura de $80{ }^{\circ} \mathrm{C}$ até a neutralização da água de lavagem. Feito isso, colocou-se as amostras em estufa com circulação de ar a $80{ }^{\circ} \mathrm{C}$ para que houvesse a evaporação do etanol não reagido. As 
reações foram realizadas em triplicata, e o valor médio reportado.

$$
\text { Conversão }(\%)=\frac{X_{a, i}-X_{a, f}}{X_{a, i}} \times 100
$$

O efeito das variáveis de processo foi avaliado por meio da ANOVA e teste estatístico de Tukey, usando o software Excel®. O percentual de ácidos graxos livres foi determinado com base no método Ca $5 \mathrm{a}-40$ onde: $X_{a, i}=$ teor de AGLs inicial no óleo e $X_{a, f}=$ teor de AGLs final na amostra do meio reacional. (AOCS, 1998) e a conversão foi extraída a partir do cálculo obtido por meio da equação 1 .

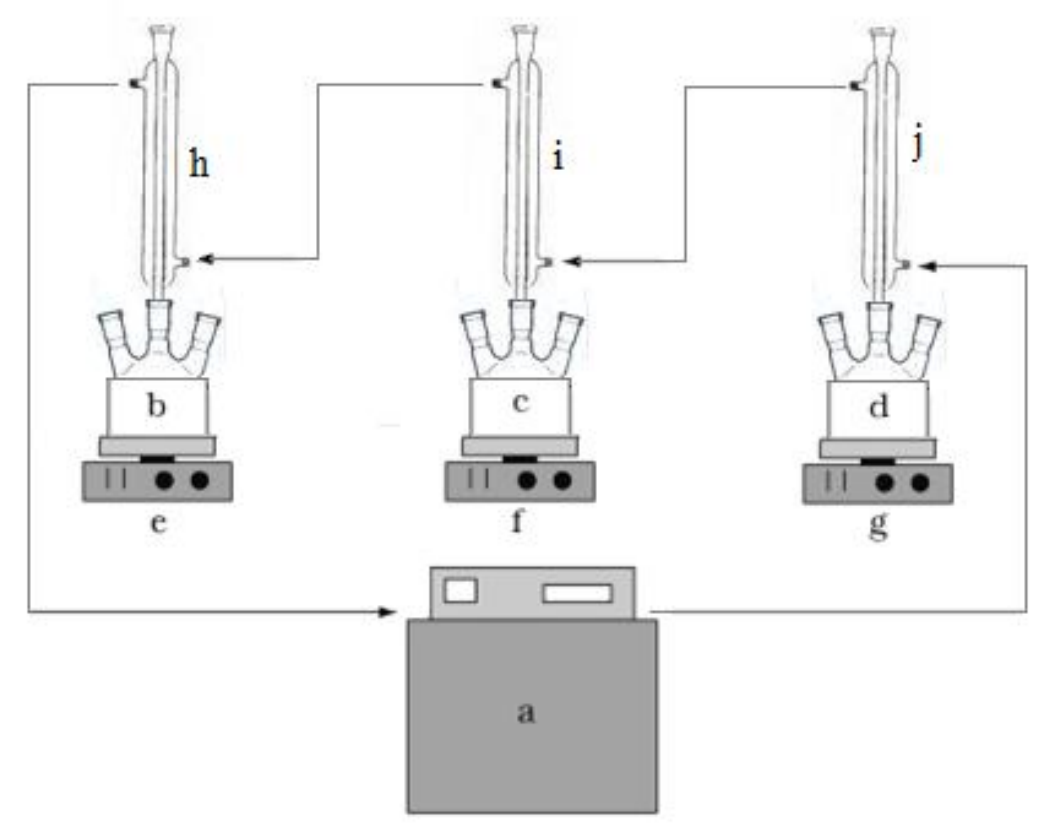

Figura 1 - Diagrama esquemático do aparato experimental utilizado para as reações de esterificação: a) banho termostatizado (Marconi); b), c) e d) balões de três bocas de vidro acoplados em mantas de aquecimento; e) f) e g) sistema de agitação e temperatura (IKA® RCT basic) e h) i) e j) condensadores.

\section{Resultados e Discussões}

\subsection{Efeito do Percentual de Catalisador}

Primeiramente, foi realizada a análise dos diferentes percentuais de catalisador $(0,5 ; 1,0 ; 1,5$ e $2,0 \%)$ sob a conversão da reação nos alcoóis etanol e metanol. A Figura 2 apresenta os resultados obtidos, onde se pode observar que 0 aumento dos teores de catalisador no meio reacional proporcionou maiores conversões de AGLs. Além disso, nota-se um comportamento semelhante em termos de conversão para os alcoóis em questão, sendo os melhores resultados obtidos para o metanol (48,17\%). Aplicando-se o teste estatístico dos dados, pode-se verificar diferença significativa $(p>0,05)$ para os diferentes percentuais de catalisador avaliados, tanto para o metanol como para o etanol, não havendo diferença para as conversões obtidas nos percentuais de 1,5 e $2,0 \%$ de $\mathrm{H}_{2} \mathrm{SO}_{4}$ de ambos os álcoois respectivamente. Maiores quantidades de catalisador no meio reacional deslocam o equilíbrio da reação, favorecendo a formação dos produtos. Mesmo efeito pode ser observado em trabalhos da literatura especializada utilizando ácido sulfúrico como 
catalisador (RAMADHAS; JAYARAJ; 2007; HANH et al., 2009; CHE et al., 2012; ZOU; LEI, MURALEEDHARAN, 2005; CHONGKONG et al., 2012).

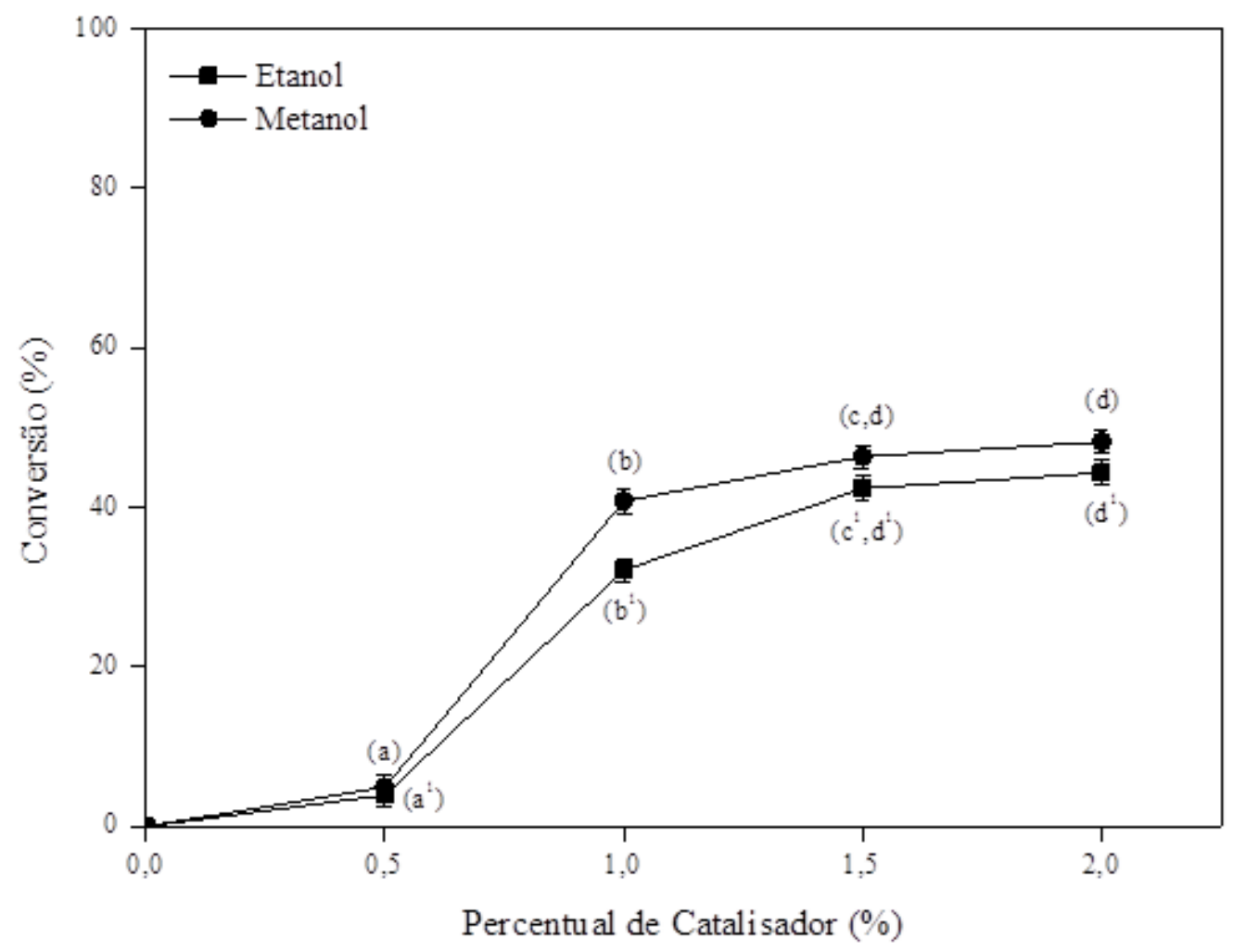

Figura 2 - Influência do percentual de catalisador e tipo de álcool na conversão da reação de esterificação dos AGLs do óleo da polpa da macaúba, sob $50 \stackrel{\circ}{\circ}$, razão molar AGLs:álcool de 1:3 e agitação de 400 rpm. Repetições de caracteres representam que não houve diferença estatística $(p>0,05)$ entre os percentuais de catalisadores utilizados.

Ramadhas, Jayaraj e Muraleedharan (2005) obtiveram aumento de $\sim 10 \%$ da conversão da reação de esterificação de AGLs ao aumentarem o percentual de catalisador $\mathrm{H}_{2} \mathrm{SO}_{4}$ de 0,4 a $0,5 \%$. Ao variar o conteúdo de $0,183-1,834 \%$ do mesmo catalisador, Chongkong et al. (2007) obtiveram maiores conversões (de 90 à $96 \%$, respectivamente) ao produzirem biodiesel via esterificação dos AGLs do destilado de óleo de palma. Che et al. (2012) verificaram maior decréscimo no conteúdo de AGLs do óleo de oliva para adição de $1 \%$ de $\mathrm{H}_{2} \mathrm{SO}_{4}$ na reação de esterificação, quando comparado a $0,5 \%$ do mesmo catalisador. Os efeitos do percentual de catalisador na eficiência da conversão de pré-esterificação do óleo de pinhão manso por meio de irradiação ultrassônica foram investigados por Zou e Lei (2012), os quais reportam conversões de $90 \%$ e $98 \%$ na utilização de $0,8 \%$ e $1,2 \%$ de $\mathrm{H}_{2} \mathrm{SO}_{4}$, respectivamente.

Os resultados obtidos podem ser comparados com a esterificação de AGLs utilizando diferentes catalisadores. Investigando os variados percentuais de catalisador na esterificação metílica dos AGLs do óleo de fritura, utilizando a resina Amberlyst ${ }^{\circledR} 15$, Gan et al. (2012), em razão molar óleo:metanol 1:15 e $65 \stackrel{\circ}{\circ}$, obtiveram conversões de $55 \%$ e $60 \%$ para utilização $1 \%$ e $4 \%$ de catalisador, respetivamente. Efeito similar 
foi observado por Dang e Chen (2013), os quais relatam o aumento da conversão do ácido palmítico em metanol com o aumento do percentual de resina Amberlite $^{\mathrm{TM}}$ IR-120.

\subsection{EfEITO dA RAZÃo MolaR AGLS: ÁlCOOL}

O efeito da razão molar AGLs:álcool foi avaliado considerando-se razões molares de 1:1 à 1:18 para o metanol e $1: 1$ à 1:12 para o etanol. Maiores quantidades de álcool no meio reacional deslocaram o equilíbrio da reação, favorecendo a conversão e formação dos produtos. A Figura 3 apresenta os resultados para as reações conduzidas sob as diferentes quantidades de álcool. Para o etanol, a maior conversão obtida ( $61 \%$ ) foi verificada em razão molar 1:9, enquanto razões superiores (1:12) ocasionaram o decréscimo na conversão da reação. O aumento inicial da razão molar provocou a diminuição da viscosidade do óleo, explicando a melhora da eficiência na conversão. No entanto, a partir da razão molar AGLs:etanol 1:6, as conversões não foram proporcionais ao aumento das razões molares. Tal fato pode ser explicado por Patil et al. (2012), os quais reportam que quantidades excessivas de álcool, ao invés de promoverem a formação dos produtos, podem reduzir a concentração do catalisador na mistura reagente e retardar a reação. Para o metanol, entretanto, reporta-se o aumento na conversão diretamente proporcional ao aumento da quantidade de álcool no meio reacional. Em razão molar AGLs:metanol de 1:1, $\sim 15 \%$ de conversão foi alcançada, enquanto que, para $1: 18$, este resultado aumentou significativamente para $\sim 93 \%$.

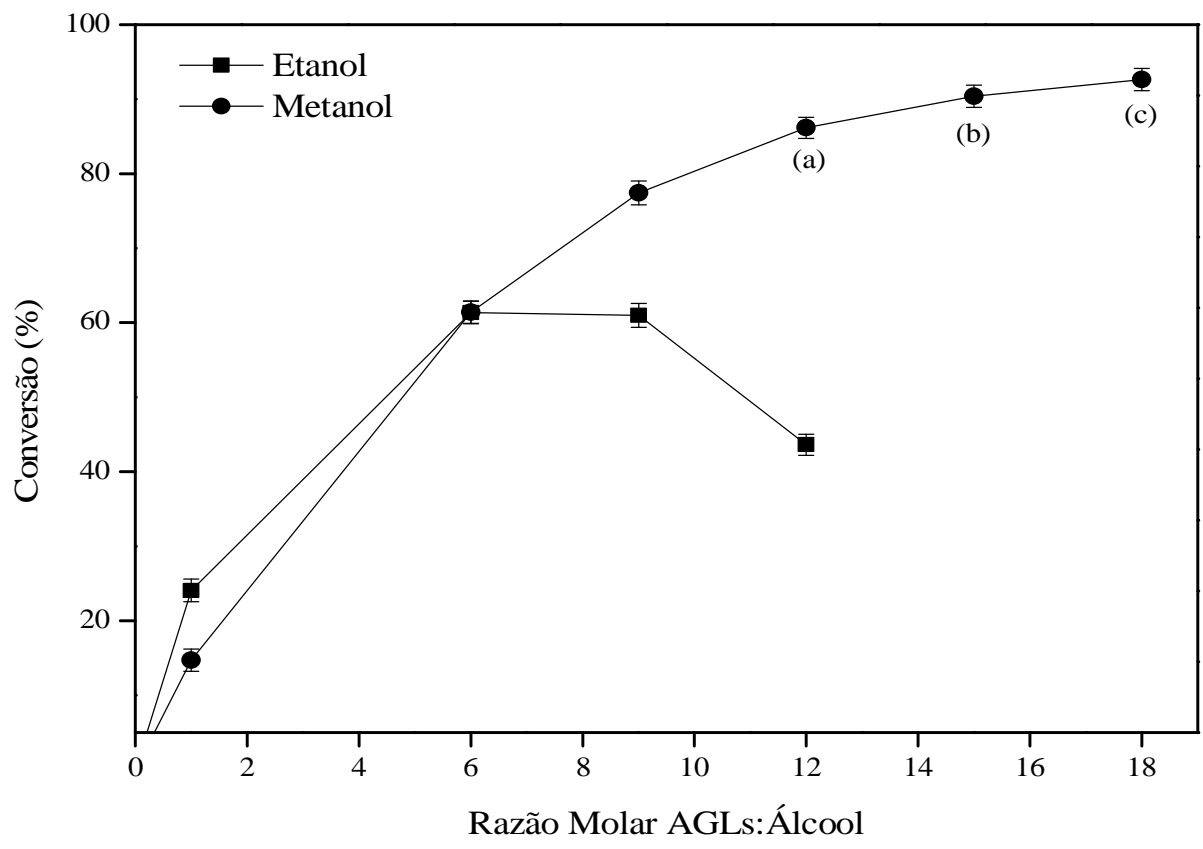

Figura 3 - Influência da razão molar na conversão da reação de esterificação dos AGLs do óleo da polpa de macaúba, sob temperatura de $50 \stackrel{\circ}{ } \mathrm{C}, 2 \%$ de catalisador $\mathrm{H}_{2} \mathrm{SO}_{4}$, agitação de $\sim 400 \mathrm{rpm}$ e tempo de reação de 1 hora. Repetições de caracteres representam que não houve diferença estatística $(p>0,05)$ entre as razões molares avaliadas.

Utilizando ácido sulfúrico como catalisador, Berrios et al. (2010) reportam o decréscimo da acidez do óleo de girassol com o aumento da razão molar ácido oleico:metanol de 1:20 a 1:80. Os autores reportam 
conversões na ordem de $85 \%$ na temperatura de 60 ${ }^{\circ} \mathrm{C}$, razão molar acido oleico:metanol de 1:80, tempo de reação de 1 hora e 5\% de catalisador (em relação à massa de ácido oleico). Realizando a esterificação homogênea ácida de ácidos graxos livres na presença de triglicerídeos, Marchetti e Errazu (2008) obtiveram conversões na ordem de $90 \%$, decorridos 250 minutos de reação, por meio de razão molar óleo:etanol (1:10), enquanto que para o mesmo período de tempo, sob razão $1: 4$, conversões de $\sim 83 \%$ foram reportadas. Trentini e Silva (2013), estudando a produção de ésteres de AGLs do óleo de caroço de algodão, em duas etapas reacionais, obtiveram na etapa de esterificação a $40{ }^{\circ} \mathrm{C}, 1,5 \%$ de catalisador $\mathrm{H}_{2} \mathrm{SO}_{4}$, durante 1 hora de reação, um aumento significativo da conversão com o aumento das razões molares de AGL:metanol 1:3 (28,95\%) para 1:9 (88,19\%). Gan et al. (2012) explicam que quando a concentração de metanol no meio reacional aumenta, o equilíbrio da reação se desloca para o lado dos produtos, resultando em uma maior conversão dos AGLs em ésteres. Além disto, ao aumentar a proporção molar do álcool em relação ao óleo, a viscosidade da mistura reacional diminui, o que, por sua vez, promove uma melhor homogeneidade entre os reagentes e o catalisador e aumenta a taxa de transferência de massa, levando finalmente a uma conversão mais elevada dentro de um tempo de reação fixo (GAN et al., 2010).

\subsection{Efeito dA Temperatura E Tempo dE REAÇÃo}

Ao ser avaliado o efeito da temperatura em relação à conversão da reação de esterificação, pode ser verificado efeito significativo na conversão dos AGLs $(p>0,05)$ nos tempos iniciais de reação, para todas as temperaturas consideradas, conforme apresentado pela Figura 4. As temperaturas de 30 e $40{ }^{\circ} \mathrm{C}$ apresentaram acentuada influência do tempo de reação. Entretanto, para as temperaturas de 50 e 60 ○C, a partir de 45 minutos de reação, não foi verificada diferença significativa $(p<0,05)$ na conversão da reação com o aumento do tempo. Decorridos 90 minutos de reação, nas temperaturas de 30 e $40{ }^{\circ} \mathrm{C}$ reporta-se conversões máximas de 62 e $82 \%$, respectivamente, enquanto conversões na ordem de $95 \%$ foram obtidas na temperatura de $60{ }^{\circ} \mathrm{C}$ e 45 minutos de reação. Em relação a este parâmetro, pode-se considerar os resultados apresentados por Charoenchaitrakool e Thienmethangkoon (2011) e por Farag, El-Maghraby e Taha (2011), em que o efeito da temperatura é similar ao relatado neste trabalho.

No trabalho de Charoenchaitrakool e Thienmethangkoon (2011), utilizando ácido sulfúrico como catalisador na esterificação de AGLs em óleos de fritura, o aumento da temperatura de $45{ }^{\circ} \mathrm{C}$ a $55^{\circ} \mathrm{C}$ favorece o rendimento em ésteres metílicos, obtendose $\sim 83 \%$ em ésteres em 1 hora de reação, razão molar óleo:metanol de 1:20, 1\% de catalisador (em relação à massa de óleo) e temperatura de $55^{\circ} \mathrm{C}$. Mesmo efeito foi verificado por Farag, El-Maghraby e Taha (2011), os quais estudaram a esterificação de AGL em uma mistura de óleo de girassol e óleo de soja utilizando acido sulfúrico como catalisador. Os autores verificaram que a conversão dos AGLs foi favorecida pelo aumento da temperatura de $30{ }^{\circ} \mathrm{C}$ a $60{ }^{\circ} \mathrm{C}$, obtendo conversões na ordem de $99 \%$ a $60 \stackrel{\circ}{\circ}$, razão molar óleo:metanol de 1:6, 2,5\% de catalisador (em relação à massa de óleo) em 1 hora de reação. 


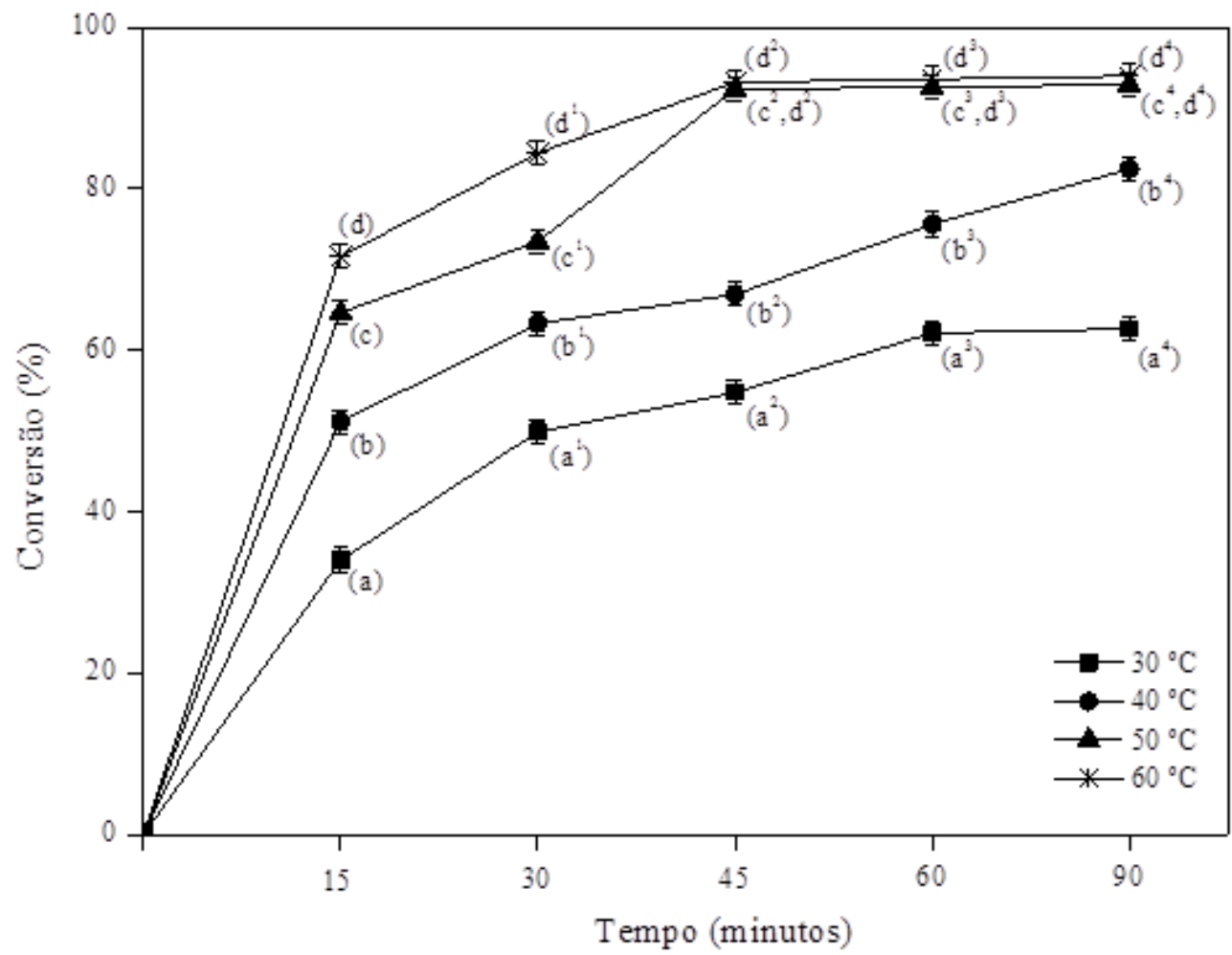

Figura 4 - Influência da temperatura na conversão reação de esterificação metílica dos AGLs do óleo da polpa de macaúba, sob razão molar óleo:metanol em 1:18, 2\% de catalisador $\mathrm{H}_{2} \mathrm{SO}_{4}$, agitação de $\sim 400 \mathrm{rpm}$ e tempo de reação de 1 hora. Repetições de caracteres representam que não houve diferença estatística $(p>0,05)$ entre as diferentes temperaturas mantendo o tempo fixo de reação.

\section{CONCLUSÕES}

A esterificação de ácidos graxos livres do óleo da polpa da macaúba utilizando o ácido sulfúrico como catalisador foi investigada. O estudo visou reportar o efeito das variáveis percentual de catalisador, razão molar AGL:álcool, temperatura e tempo de reação na conversão dos AGLs. Os resultados reportam que o aumento do percentual de catalisador $0,5-1,5 \%$ (em relação à massa de $\mathrm{AGL}$ ) favorece a reação. Conversões na ordem de $93 \%$ puderam ser obtidas em razões molares 1:18 óleo:metanol, enquanto que o máximo obtido para o etanol foi de $\sim 61 \%$, em razão molar 1:9, ambos em tempo de reação de 1 hora. Em relação à temperatura, aumento significativo na conversão pôde ser observado, com o aumento da temperatura reacional na faixa de $30{ }^{\circ} \mathrm{C}$ a $60 \stackrel{\circ}{\circ} \mathrm{C}$.
Conversões de $\sim 95 \%$ puderam ser obtidas para temperatura de $50 \stackrel{\circ}{\circ}$, sob razão molar óleo:metanol em 1:18, 2\% de catalisador, agitação de 400 rpm e tempo de reação de 1 hora. Os resultados obtidos demonstram que altas conversões dos AGLs do óleo da polpa da macaúba podem ser obtidas utilizando o ácido sulfúrico como catalisador, possibilitando a sua utilização para a etapa posterior de transesterificação dos triglicerídeos.

\section{AgRADECIMENTOS}

Os autores agradecem à Fundação Araucária pelo suporte financeiro, à CAPES pela concessão da bolsa e à Universidade Estadual de Maringá - Campus Regional de Umuarama - pela infraestrutura. 


\section{REFERÊNCIAS}

ABREU, I. S. et al. First karyotype, DNA C-value and AT/GC base composition of macaw palm (Acrocomia aculeata) - a promising plant for biodiesel production. Australian Journal of Botany, 59(2), 149-155, 2011.

AOCS - Official Methods and Recommended Practices of the American Oil Chemists' Society (Method AOCS Ca 5a-40), 5th ed., edited by WALKER, R. E. American Oil Chemists' Society, Champaign, 1998.

BERRIOS, M. et al. Study of esterification and transesterification in biodiesel production from used frying oils in a closed system. Chemical Engineering Journal, 160(2), 473-479, 2010.

CHAROENCHAITRAKOOL, M.; THIENMETHANGKOON, J. Statistical optimization for biodiesel production from waste frying oil through twostep catalyzed process. Fuel Processing Technology, 92(1), 112-118. 2011.

$\mathrm{CHE}, \mathrm{F}$. et al. Exploring a promising feedstock for biodiesel production in Mediterranean countries: A study on free fatty acid esterification of olive pomace oil. Biomass and Bioenergy, 36, 427-431, 2012.

CHONGKONG, S. et al. Biodiesel production by esterification of palm fatty acid distillate. Biomass and Bioenergy, 31(8), 563-568, 2007.

CORRO, G. et al. Biodiesel from waste frying oil. Two step process using acidified $\mathrm{SiO} 2$ for esterification step. Catalysis Today, 166(1), 116-122, 2011.

DANG, T. H.; CHEN, B. H. Optimization in esterification of palmitic acid with excess methanol by solid. Fuel Processing Technology, 109, p. 7-12, 2013.

DI SERIO, M. et al. Heterogeneous catalysts for biodiesel production. Energy \& Fuels, 22(1), 207-217, 2008.

FARAG, H. A.; EL-MAGHRABY, A.; TAHA, N. A. Optimization of factors affecting esterification of mixed oil with high percentage of free fatty acid. Fuel Processing Technology, 92(3), 507-510, 2011.

GAN, S. et al. Ferric sulphate catalysed esterification of free fatty acids in waste cooking oil. Bioresource Technology, 101(19), 7338-7343, 2010.

GAN, S. et al. Heterogeneous free fatty acids esterification in waste cooking oil using ion-exchange resins. Fuel Processing Technology, 102, 67-72, 2012.

HANH, H. D. et al. Biodiesel production by esterification of oleic acid with short-chain alcohols under ultrasonic irradiation condition. Renewable Energy, 34(3), 780-783, 2009.

LOTERO, E. et al. Synthesis of biodiesel via acid catalysis. Industrual \& Engineering Chemistry Research, 44, 5353-5363, 2005.

LOU, W. Y.; ZONG, M. H.; DUAN, Z. Q. Efficient production of biodiesel from high free fatty acidcontaining waste oils using various carbohydratederived solid acid catalysts. Bioresource Technology, 99(18), 8752-8758, 2008.

MA, F.; HANNA, M. Biodiesel production: a review. Bioresource Technology, 70(1), 1-15, 1999.

MARCHETTI, J. M.; MIGUEL, V. U.; ERRAZU, A. F. Possible methods for biodiesel production. Renewable \& Sustainable Energy Reviews, 11(6), 1300-1311, 2007.

MARCHETTI, J. M.; ERRAZU, A. F. Comparison of different heterogeneous catalysts and different alcohols for the esterification reaction of oleic acid. Fuel, 87(15-16), 3477-3480, 2008.

MBARAKA, I. K. et al. Organosulfonic acidfunctionalized mesoporous silicas for the esterification of fatty acid. Journal of Catalysis, 219(2), 329-336, 2003.

McCORNICK, R. L. et al. Impact of Biodiesel Source Material and Chemical Structure on Emissions of Criteria Pollutantes from a Heavy-duty Engine. Environmental Science and Engineering, 35, 1742, 2001.

MINAMI, E.; SAKA, S. Kinetics of hydrolysis and methyl esterification for biodiesel production in twostep supercritical methanol process. Fuel, 85(17-18), 2479-2483, 2006.

NG, H. K.; GAN, S. Combustion performance and exhaust emissions from the nonpressurised combustion of palm oil biodiesel blends. Applied Thermal Engineering, 30(16), 2476-2484, 2010.

PATIL, P. D. et al. Biodiesel Production from Waste Cooking Oil Using Sulfuric Acid and Microwave Irradiation Processes. Journal of Environmental Protection, 3, 107-113, 2012. 
RAMADHAS, A. S.; JAYARAJ, S.; MURALEEDHARAN, C. Biodiesel production from high FFA rubber seed oil. Fuel, 84(4), p. 335-340, 2005.

RAMOS, L. P. et al. Biodiesel: Um projeto de sustentabilidade econômica e sócio-ambiental para o Brasil. Biotecnologia Ciência \& Desenvolvimento, 31, 27-37, 2003.

ROCHA, L. L. L. et al. Production of Biodiesel by a Two-Step Niobium Oxide Catalyzed Hydrolysis and Esterification. Letters in Organic Chemistry, 7(7), 571-578, 2010.

SOUSA, J. S. et al. Application of lipase from the physic nut (Jatropha curcas L.) to a new hybrid (enzyme/chemical) hydroesterification process for biodiesel production. Journal of Molecular Catalysis B, Enzymatic, 65(1-4), 133-137, 2010.

SRILATHA, K. et al. Efficient Esterification and Transesterification of Used Cooking Oil Using 12Tungstophosphoric Acid (TPA)/ $\mathrm{Nb}_{2} \mathrm{O}_{5}$ Catalyst. Energy \& Fuels, 24(9), 4748-4755, 2010.

SUAREZ, P. A. Z. et al. Transformação de triglicerídeos em combustíveis, materiais poliméricos e insumos químicos: algumas aplicações da catálise na oleoquímica. Química Nova, 30(3), 667-676, 2007.
TALUKDER, M. M. R.; WU, J. C.; CHUA, L. P. L. Conversion of Waste Cooking Oil to Biodiesel via Enzymatic Hydrolysis Followed by Chemical Esterification. Energy \& Fuels, 24(3), 2016-2019, 2010.

SILVA, C.; TRENTINI, C. P. Produção de ésteres de ácidos graxos a partir do óleo de caroço de algodão em duas etapas reacionais. Engevista (UFF), 15(3), 235-242, 2013.

VYAS, A. P.; VERMA, J. L.; SUBRAHMANYAM, N. A review on FAME production processes. Fuel, 89(1), 19, 2010.

ZOU, H.; LEI, M. Optimum process and kinetic study of Jatropha curcas oil pre-esterification in ultrasonical field. Journal of the Taiwan Institute of Chemical Engineers, 43(5), 730-735, 2012.

ZULLAIKAH, S. et al. A two-step acid-catalyzed process for the production of biodiesel from rice bran oil. Bioresource Technology, 96(17), 1889-1896, 2005 . 